\title{
A revisão como um instrumento para a melhoria da qualidade de textos
}

\section{narrativos}

\section{The revision as an instrument for improving the quality of narrative texts}

\author{
Sandra Borges, Ana Cristina Silva \\ ISPA-IU
}

\begin{abstract}
Resumo
O objetivo deste estudo é o de avaliar o impacto na escrita de textos narrativos, de um programa de escrita, em que os alunos têm acesso a instrumentos de autorregulação para a revisão dos textos. Quisemos perceber se o recurso à utilização de grelhas e ao feedback do professor influenciava a qualidade dos textos escritos por parte dos alunos. Participaram 71 crianças do $4^{\circ}$ ano de escolaridade, divididas em 3 grupos experimentais, (submetidas a um programa de intervenção) e 1 de controlo. Os resultados demonstraram que os alunos que utilizaram as grelhas e receberam o feedback aumentaram a qualidade dos seus textos.

Palavras chave: produção de narrativas, revisão, aprendizagem autorregulada, crianças do primeiro ciclo.
\end{abstract}

\begin{abstract}
The purpose of this research was to evaluate the impact of a writing program, involving a narrative text revision with a rubric. The participants were 71 children from 4 th grade divided in 3 Experimental groups (submitted to an intervention program), and 1 Control Group. The effect of a training program was evaluated, in which children revised their own texts with the help of a rubric. We wanted to see if the use of a rubric and teacher's feedback influenced the quality of the written texts written by the students. Our results showed an improvement in the quality of 4th grade children's written composition from the Experimental Group in comparison to the Control Group.

Keywords: narrative production, revision, self-regulation, primary education
\end{abstract}

A aprendizagem da escrita é um processo longo, complexo e cognitivamente exigente, devido à complexidade e multiplicidade dos seus objetivos (Limpo, 2014). O caminho para a proficiência na escrita é um objetivo a ter em conta desde a entrada dos alunos no primeiro ano de escolaridade e dela depende o sucesso das aprendizagens escolares nas fases posteriores.

A produção textual implica a utilização de várias competências, tais como a produção rápida e precisa de letras e palavras, geração de ideias, seleção de palavras, uso apropriado de gramática e pontuação, uma ortografia exata, planeamento, tradução do planeamento para sequências de linguagem, avaliação e revisão
(Alamargot \& Fayol, 2009; Galbraith, 2006; Hammill, 1987; Graham, 1990 \& Torrance, 2006). Os alunos devem, portanto, conhecer as regras sintáticas que regulamentam a língua portuguesa (Hayes, 2006; Mateus, Pereira \& Fisher, 2008; Pinheiro, 2007).

Após o mecanismo das correspondências grafo-fonéticas estar adquirido, a instrução sobre a escrita passa a incidir sobre o desenvolvimento de competências mais avançadas como produzir frases complexas, planear a escrita e rever o que se escreveu, capacidades necessárias à produção de textos (Berninger, Mizokawa \& Bragg, 1991; Gersten \& Baker, 2001; Limpo \& Alves, 2013).

Segundo Hayes e Flower (1986), os objetivos da escrita podem organizar-se de forma hierárquica, envolvendo três processos cognitivos centrais que se inter-relacionam: a planificação, a textualização e a revisão. Planear refere-se às ideias prévias que os escritores possuem sobre o que pretende transmitir (Hayes \& Flower, 1980). Esta fase é acompanhada de processos relacionados com os objetivos a atingir com o texto, que podem ou não ser percetíveis. Para o escritor poder escrever o que tem planeado, necessita de transformar as representações linguísticas que operam na sua memória de trabalho, que por sua vez se traduzem em frases, para depois constituírem um texto.

A revisão significa fazer qualquer mudança em qualquer momento da composição escrita. É um processo cognitivo de resolução de problemas que envolve a deteção de discrepâncias entre os textos pretendido e realizado, decisões sobre como fazer as mudanças desejadas e realizar essas mudanças.

As mudanças podem ou não afetar o significado do texto, e podem ser grandes ou pequenas. Note-se também que, as mudanças podem ser feitas na mente do escritor antes do texto ser escrito no papel, enquanto o texto está a ser escrito, e/ou depois do texto ter sido escrito. Fitzgerald (1987, p. 484).

Vários são os estudos que demonstram que para se desenvolver uma boa competência no processo da escrita não basta possuirmos somente habilidades mecânicas, teremos que desenvolver altos níveis de autorregulação (Graham \& Harris, 2000; Fidalgo \& García, 2008; (Montanero, Lucero \& Fernández, 2014; 
Redondo, Torrance \& Ramón, 2011), que envolvem um bom conhecimento do processo de escrita e um domínio autorregulado e estratégico dos referidos processos cognitivos. Os aspetos mencionados evidenciam duas dimensões da metacognição, o autoconhecimento e a autorregulação, que estão presentes em todas as tarefas que desempenhamos.

A metacognição nas tarefas de escrita refere-se à tomada de consciência, por parte do escritor, dos conhecimentos que possui relativamente ao propósito, ao processo, à audiência e à autorregulação da escrita (Garcia \& Herreras, 2004). Vários investigadores consideram que os alunos devem escrever frequentemente (Graham, Gillespie \& Mckeown, 2013; Graham, Harris \& Herbert, 2011; Rogers \& Graham, 2008), mas também referem que as estratégias de autorregulação no processo de aprendizagem necessitam de ser adquiridas (Fidalgo \& Garcia, 2008; Zimmerman \& Reisemberg, 1997).

O processo de revisão da escrita tem como principais funções detetar e corrigir incoerências no discurso escrito, ao nível das convenções linguísticas e do seu significado, bem como avaliar se o discurso escrito se encontra conforme os objetivos traçados pelo escritor (Hayes \& Flower, 1980, 1986). Deste modo ao rever um texto o escritor está a avaliar um conjunto de ações que efetuou em momentos anteriores e que irão influenciar tanto os aspetos estruturais, como os aspetos superficiais do texto, fatores estes que se irão repercutir na qualidade do mesmo (Englert, Raphael, Anderson, Stevens \& Fear, 1991).

São vários os estudos que referem os benefícios da autorregulação na escrita, assim como as melhorias efetuadas nos textos após a realização do referido processo (Montanero et al., 2014; Limpo, 2014; Schunk \& Zimmerman, 2007; Silva, 2013).

Segundo Leitão e Ribeiro (2009) citando (Alamargot \& Chanquoy, 2001) a escrita de textos é comparada à resolução de problemas a serem solucionados pelo autor.

Outros estudos referem que a diferença de idades e o avanço na escolaridade são fatores que interferem na melhoria da revisão (Graham \& Harris, 2000; Limpo, 2014; Silva, 2013).

Tem-se verificado que os alunos do Ensino Básico, em especial os do $1^{\circ}$ ciclo, escrevem os seus textos sem refletirem sobre os seus conteúdos ou estrutura, sem os reverem, sem solucionarem os problemas que os referidos textos apresentam (Barbeiro \& Pereira, no prelo; Scardamalia \& Bereiter, 1987).

Silva, citando (Hayes, 2000; Lahey \& Bloom, 1994) considera que as crianças mais novas apresentam capacidades de revisão mais limitadas pois revelam dificuldades em estabelecer objetivos claros em relação à escrita, revelam igualmente dificuldades na avaliação da sua própria escrita, descurando que os seus textos irão ser lidos por outrem. Revelam igualmente dificuldades em analisar as mudanças que devem realizar nos seus produtos escritos e em proceder a essas mudanças e por vezes não possuem capacidades executivas para resolver problemas que surgem no ato de rever (Fitzgerald, 1987). Estudos semelhantes com crianças dos mesmos níveis de ensino demonstraram que após terem sido ensinadas às crianças estratégias de planeamento e escrita de textos, bem como a utilização de procedimentos para autorregulação dos seus processos de escrita, os mesmos melhoraram significativamente, quando comparados às crianças do grupo de controlo. (Gundín \& Sánchez, 2008; Limpo \& Alves, 2013; Montanero, Lucero \& Fernández 2014; Silva, 2013; Zimmerman, 2002).

Segundo Pereira (2000), o professor deve orientar os alunos para o processo de revisão, transmitindo-lhes os conhecimentos necessários para que os alunos possam construir o seu próprio conhecimento. Devido ao facto de os alunos não possuírem conhecimentos de organização textual, torna-se fundamental a definição de critérios de avaliação semelhantes aos objetivos pedagógicos do docente.

Montanero et al., (2014), desenvolveram um estudo com crianças do Ensino Básico, cujo objetivo era o de recolher informação sobre a utilização de uma grelha de apoio à revisão, constituída por sete critérios e quatro níveis de execução. Os resultados demonstraram que os alunos que efetuaram a revisão com recurso à grelha apresentaram melhores resultados na organização da estrutura das suas narrativas.

O termo feedback é definido como sendo a ajuda, prestada pelo professor aos alunos, quando estes sentem dificuldades numa determinada tarefa (Nicole \& Macfarlane, 2006). O feedback escrito refere-se aos comentários efetuados pelo professor relativamente às produções escritas de um aluno. Os referidos comentários centram-se nos critérios de avaliação definidos pelo professor relativamente à tarefa a desenvolver (Rust, Price \& Donovan, (2003). É, no entanto, importante referir que o feedback deve ser fornecido ao aluno preferencialmente na primeira versão do seu produto escrito, pois a apreciação da primeira versão irá desenvolver no aluno a possibilidade de construção de novas aprendizagens (Bloxham \& Campbell, 2010; Dias \& Santos 2013).

Tanto professores como alunos consideraram o feedback escrito como uma parte fundamental no processo da escrita (Cohen \& Cavalcanti, 1990; Fathman \& Whalley, 1990; Ferris, 1995).

Um processo de revisão eficaz em textos narrativos implica a tomada de consciência das dimensões da estrutura narrativa. Segundo Marques \& Ferreira, (2006), os textos são identificáveis segundo a sua sequência de etapas ou passos funcionais distintos, no entanto, devem obedecer a uma sequência definida, a sua estrutura. $O$ texto narrativo desenvolve-se em função daquilo que podemos referir como a sua estrutura de género. No caso do género narrativo de acontecimentos, tem-se como adquirido que do potencial de estrutura genológica fazem parte os seguintes elementos obrigatórios: Localização Temporal Inicial, Localização Espacial Inicial, Introdução de Personagens e Sequencialização Lógica de Eventos; também pode ocorrer, opcionalmente, um Fechamento da Narrativa, (Mateus, Pereira \& Fischer, 2008). 


\section{Método}

\section{Participantes}

Participaram 71 crianças do $4^{\circ}$ ano de escolaridade com práticas semelhantes ao nível do trabalho sobre o texto escrito na sala de aula, nas quais as instruções específicas sobre a estrutura do texto narrativo eram pouco frequentes. Estas crianças foram divididas em 3 grupos experimentais, (submetidas a um programa de intervenção) e 1 de controlo, tendo sido controladas as variáveis relativas ao nível cognitivo e ao desenvolvimento da linguagem ao nível lexical e sintático.

Os grupos eram estatisticamente equivalentes quanto ao nível intelectual, desenvolvimento lexical e compreensão de estruturas complexas. Foi utilizado o teste Kruskal-Wallis, mostrando que os mesmos não diferiam, nestas dimensões no início da intervenção ( $p>$ $.05)$.

A recolha de dados foi organizada em duas fases. $\mathrm{O}$ pré-teste, que consiste na produção de um texto por parte dos alunos do $4^{\circ}$ e um pós-teste em que se efetuou o mesmo procedimento. Entre o pré e o pós-teste foi efetuada a intervenção experimental. A intervenção experimental diferiu em função dos grupos:

Grupo experimental 1- Utilização da grelha para autorregulação e sem feedback do professor (o feedback consiste em indicações ao aluno sobre o resultado do seu desempenho na escrita dos textos, indicando especificamente a natureza das falhas no seu texto.

Grupo experimental 2- Utilização da grelha para autorregulação e com feedback do professor (o feedback consiste no retorno ao aluno sobre o resultado do seu desempenho na escrita dos textos. $\mathrm{O}$ docente assinala na grelha do aluno as dimensões que o aluno não referiu na sua narrativa, ou seja, as dimensões (personagens, narrativa, etc.,) e que deverá melhorar quando o aluno reescrever o seu texto).

Grupo experimental 3- É pedido ao aluno para rever o texto e melhorá-lo reescrevendo-o, sem que, no entanto, lhe sejam proporcionadas indicações sobre como o fazer ou em que dimensões o deverá melhorar. Este grupo não possui grelha nem feedback do professor. E finalmente o Grupo de controlo que efetuou exercícios de matemática.

\section{Instrumentos}

\section{Grelha para avaliação dos textos}

Para se analisar e posteriormente cotar os textos narrativos elaborados pelos alunos, foi construído um modelo de análise, um suporte estruturado em grelha, com base nos critérios definidos na prova PROESC (Cuetos, Ramos \& Ruano, 2002) e no modelo de análise de narrativas escritas do estudo sobre a Diversidade Linguística na Escola Portuguesa, desenvolvido pelo ILTEC (Mateus et al., 2008). O referido modelo contempla os seguintes itens: Introdução, Descrição das personagens, Acontecimentos e seu desenlace, Desenlace coerente, Criatividade, Continuidade lógica, Sentido global do texto, Sinais de pontuação, Frases completas e Vocabulário. Foi atribuída uma cotação a cada um dos 10 níveis de análise da narrativa, sendo a cotação máxima de 9,5 pontos. Atribuíram-se 9,5 pontos em 10 níveis, devido ao facto de na segunda dimensão, descrição das personagens, que é composta por quatro critérios, somente foi pontuado um dos critérios consoante o tipo de descrição efetuada pelo aluno. De acordo com o manual da prova, considerou-se que cada um destes critérios contava com dois níveis de desempenho aos quais aos quais se atribuiu uma determinada pontuação. Deste modo a pontuação " 0 " corresponde à ausência dos requisitos desse critério no texto avaliado, enquanto a pontuação " 1 " serviu para indicar a sua presença. A pontuação total máxima que cada aluno podia obter, somando todos os critérios era de 9,5 pontos. A grelha para avaliar os textos narrativos é uma escala descritivo-ordinal, de enfoque analítico. Como se trata de uma prova espanhola e o âmbito do nosso estudo é a Língua Portuguesa, tornou-se necessário a sua adaptação.

A PROESC é uma bateria de avaliação dos processos de escrita (Cuetos et al., 2002) composta por 10 critérios de avaliação agrupados em duas grandes dimensões: conteúdos e coerência. A dimensão "Conteúdos "baseia-se nos seguintes critérios: onde e quando, personagens, sucesso, consequências, desenlace coerente e criatividade. A dimensão "Coerência-estilo" engloba não só a coerência entre as ideias do relato, mas também a sua construção gramatical e lexical, com os seguintes critérios: continuidade lógica, sentido unitário, figuras literárias, orações complexas e vocabulário. $\mathrm{O}$ manual do instrumento informa de uma consistência interna de 0,82 (coeficiente de alfa). Apresenta também, uma boa validade, referida a critério e uma adequada validez fatorial.

\section{Grelha para revisão dos textos}

A grelha para a revisão dos textos, por parte dos alunos, foi um instrumento criado ad hoc para esta investigação. O material de apoio à revisão do texto, que denominamos de grelha, consistiu num simples guião de perguntas agrupadas, tendo em conta as partes que compõem uma narrativa. As perguntas focalizam-se na estrutura da narrativa baseada na prova (PROESC) Prova de Avaliação dos Processos de Escrita, (Cuetos et al., 2002) e no modelo de análise de narrativas escritas do estudo sobre a Diversidade Linguística na Escola Portuguesa, desenvolvido pelo ILTEC (Mateus et al., 2008).

\section{Procedimento}

Esta investigação obedeceu a design experimental com um pré-teste e pós-teste onde era pedido às crianças para escreverem uma narrativa com base numa sequência de imagens. O programa de intervenção foi composto por 11 sessões com uma hora de duração cada. Entre a sessão 2 e a sessão 17 os alunos efetuaram sempre os mesmos procedimentos. As crianças dos grupos experimentais escreviam uma composição, sempre com base numa sequência de imagens e reviam a sua versão inicial. Às crianças dos grupos experimentais 1 e 2 era-lhes proporcionado uma grelha com indicações sobre os principais elementos de uma 
estrutura narrativa, com base na qual deveriam orientar a sua revisão. Em relação às crianças do grupo experimental 2 era-lhes explicitado, na grelha que possuíam para reverem as suas narrativas, através de um feedback escrito, os elementos em que falharam no seu texto, de modo a poderem melhorá-lo, enquanto que com os sujeitos do grupo experimental 1 isso não acontecia. Às crianças do grupo experimental 3 era-lhes apenas pedido que revissem os textos sem recurso a qualquer tipo de instrumento e indicação específica. As crianças do grupo de controlo despendiam o mesmo tempo em aulas de matemática.

Estas composições foram pontuadas por dois observadores de acordo com os critérios definidos na prova PROESC (Cuetos et al., 2002).
O pós-teste foi efetuado 11 semanas após a intervenção, seguindo-se o mesmo procedimento efetuado na avaliação inicial.

\section{Resultados}

Os resultados apontam para uma evolução significativa em relação à qualidade dos textos narrativos produzidos entre o pré e o pós- teste por parte de todos os grupos experimentais, o mesmo não acontecendo com o grupo de controlo. Essa evolução foi estatisticamente superior no grupo experimental 2 quando comparada aos outros grupos experimentais, verificando-se ainda que o grupo experimental 1 foi estatisticamente superior ao grupo experimental 3 .

Tabela 7.

Evolução pré-teste e pós-teste $-4^{\circ}$ ano

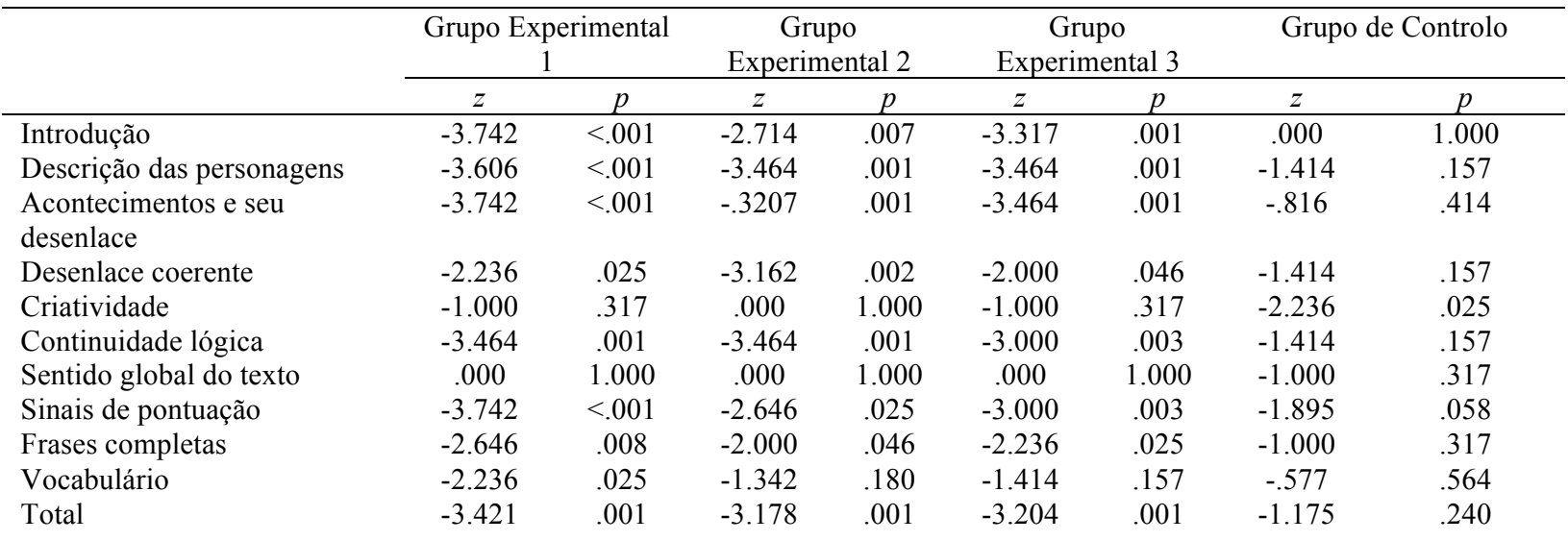

\section{Discussão}

No presente estudo pretendíamos investigar o impacto de instruções orientadas para o processo de revisão, na escrita de narrativas, através da utilização de grelhas em crianças do $4^{\circ}$ ano de escolaridade.

Sabemos que uma das principais dificuldades apresentadas pelos alunos destas idades está relacionada com a estrutura dos textos (Gallego, 2012; Ramos, Cuadrado, \& Iglesias, 2005).

Foram encontradas diferenças estatisticamente significativas entre o Grupo de controlo e o Grupo experimental 2, ao nível da diferença entre pré-teste e pós-teste para a Introdução, Descrição das personagens, Acontecimentos e seu desenlace, Desenlace coerente, Criatividade, Continuidade lógica e Total, onde o grupo experimental 2 apresentou valores superiores aos do grupo de controlo, com exceção da Criatividade que foi superior no grupo de controlo. Concluímos assim que a qualidade das produções textuais dos alunos que foram sujeitos a um programa de escrita, onde tiveram acesso a instrumentos de autorregulação e onde lhes foi proporcionado feedback para efetuarem a revisão dos seus textos, foi superior à qualidade dos textos dos alunos que fizeram a revisão com os referidos instrumentos, sem lhes ter sido proporcionado feedback.

Estudos semelhantes com crianças dos mesmos níveis de ensino demonstraram que após terem sido ensinadas às crianças estratégias de planeamento e escrita de textos,

bem como a utilização de procedimentos para autorregulação dos seus processos de escrita, os mesmos melhoraram significativamente, quando comparados às crianças do grupo de controlo (Montanero et al. 2014; Silva, 2013).

Podemos igualmente verificar que a qualidade dos textos dos alunos que participaram no programa de escrita é superior à qualidade dos textos dos alunos que não participaram no programa, pois foram encontradas diferenças estatisticamente significativas entre os grupos para todas as dimensões exceto Criatividade, Sentido global do texto, Sinais de pontuação e Vocabulário. Os resultados mostram que nos pós-teste o grupo de controlo tem os resultados mais baixos em todas as dimensões com diferenças estatisticamente significativas entre os grupos (todas as dimensões exceto Criatividade, Sentido global do texto, Sinais de pontuação e Vocabulário). O grupo sem feedback apresenta valores superiores nas dimensões Introdução, Descrição das personagens, Frases completas e Total. O grupo com feedback apresenta valores superiores nas dimensões Acontecimentos e seu desenlace, Desenlace coerente e Continuidade lógica. Vários estudos concluem que um programa efetivo de escrita deve incluir o ensino explícito dos passos principais do 
processo de escrita (planificação, textualização e revisão), o ensino das dimensões críticas dos vários tipos de texto e estruturas que forneçam feedback aos alunos sobre o seu trabalho (Fayol, 2007; Panadero \& Jonsson, 2013; Pritchard \& Honeycutt, 2006).

Os resultados obtidos na presente investigação consideram-se promissores, pelo que sería benéfico a sua aplicação em contexto de sala de aula. Em Portugal é relativamente escasso o trabalho pedagógico, orientado para o proceso de revisão da escrita, com recurso a instrumentos de autorregulação, privilegiando-se somente o produto final e não o processo.

\section{Referências}

Alamargot, D., \& Fayol, M. (2009). Modeling the development of written transcription. In R. Beard, D. Myhill, M. Nystrand, \& J. Riley (Eds.), Handbook of Writing development (pp.23-47). London:Sage.

Arias-Gundín \& García-Sánchez, (2008). Implicaciones educativas de los modelos teóricos del proceso de revisión textual. Papeles del Psicólogo, 29(2).

Barbeiro, L. B., \& Pereira, L. A. (no prelo). O ensino da escrita: a dimensão textual. Lisboa: Ministério da Educação.

Berninger, V. W., Mizokawa, D. T., \& Bragg, R. (1991). Theory-based diagnosis and remediation of writing disabilities. Journal of School Psychology, 29, 57-79

Bloxham, S., \& Campbell, L. (2010). Generating dialogue in assessment feedback: Exploring the use of interactive cover sheets. Assessment Evaluation in Higher Education, 35(3), 291-300. doi.org/10.1080/02602931003650045

Cohen, A. D. \& Cavalcanti, M. C. (1990). Feedback on compositions: Teacher and student verbal reports.In B. Kroll (Ed.), Second language writing: Research insights

Cuetos, F., Ramos, J. L., \& Ruano, E. (2002). PROESC. Evaluación de los procesos de escritura. Madrid: TEA.

Dias, P., \& Santos, L. (2013).Práticas Avaliativas para promoção da autorregulação da aprendizagem matemática: O feedback escrito em relatórios escritos em duas fases. Quadrante. Vol. XXII.

Englert, C., Raphael, T., Anderson, L., Anthony, H.\& Stevens, D. Fear. (1991). Making writing strategies in and self-talk visible: Cognitive strategy instruction writing in regular and special education classrooms. American Educational Research Journal, 28, 337-373.

Fayol, M. (2004). Text and cognition. In Handbook of children's literacy (pp. 181-197). Springer Netherlands. doi: 10.1007/978-94-017-1731-1_10

Fayol, M. (2007). La production de textes et son apprentissage. Écrire des textes, 1 'apprentissage et le plaisir, 21.

Fitzgerald, J. (1987). Research on revision in writing. Review of Educational Research, 57, 481-506

Fidalgo, R., \& García, J. N. (2008). El desarrollo de la competencia escrita a través de una enseñanza metacognitiva de la escritura. Cultura y Educación,
20(3),

$325-346$. doi.org/10.1174/113564008785826321

Fathman, A., \& Whalley, E. (1990). Teacher response to student writing: Focus on form versus content. In B. Kroll (Ed.), Second language writing: Research insights for the classroom (pp. 178-190). New York: Cambridge University Press.

Ferris, D. R. (1995). Student reaction to teacher response in multiple-draft composition classrooms. TESOL Quarterly, 29.1, 33-53.

Galbraith, P., \& Stillman, G. (2006). A framework for identifying student blockages during transitions in the modelling process. ZDM, 38(2), 143-162. doi:10.1007/BF02655886

Graham, S. (1990). The role of production factors in learning disabled students' compositions. Journal of Educational Psychology, 82, 781-791

Graham, S., \& Harris, K. (2000). The role of self-regulation and transcription skills in writing and writing development. Educational Psycologist, 35(1), 3-12. doi.org/10.1207/S15326985EP3501_2

Gersten, R., \& Baker, S. (2001). Teaching expressive writing to students with learning disabilities: A meta-analysis. Elementary School Journal, 101, 251-272

Hayes, J. e L. Flower (1986). "Writing research and the writer". American psychologist, 41(10), 1106-1113

Hayes, J. R. (2000). A new framework for understanding cognition and affect in writing. In $\mathrm{R}$. Indrisano \& J. R. Squire (Eds.), Perspectives on writing: Research, theory and practice (pp. 6-44). Newark, DE: International Reading Association

Hayes, J. R., \& Flower, L. S. (1980). Identifying the organization of the writing process. In L. W. Gregg \& E. R. Steinberg (Eds.), Cognitive processes in writing (pp. 3-30). Hillsdale, NJ: Erlbaum

Hayes, J. (2006). New Directions in Writing Theory. In.: Macarthur, Ca Graham, S. Fitzgerald, J.(Org.). Handbook of Writing Research.

Hammill, D. (1987). Assessing the abilities and instructional needs of students. In D. D. Hammill (Ed.), Assessing the abilities and instructional needs of students: A practical guide for educators, psychologists, speech pathologists, and diagnosticians (pp. 5-37). Austin, TX: Pro-Ed

Lahey, M., \& Bloom, L. (1994). Variability and language learning disabilities. In G. P. Wallach \& K. G. Butler (Eds.), Language, learning disabilities in school-age children and adolescents: Some principles and applications (pp. 354-372). Needham Heights, MA: Allyn \& Bacon

Leitão, C., \& Ribeiro, I. (2009). Escrita de textos no quarto ano de escolaridade: Desenvolvimento e implementação de um programa de intervenção. In Actas do X Congresso Internacional GalegoPortuguês de Psicopedagogia.

Limpo, T., Alves, R. A., \& Fidalgo, R. (2014). Children's high level writing skills: Development of planning and revising and their contribution to writing quality. British Journal of Educational Psychology, 84(2), 177-193. 
Marques, C., Silva, I., \& Ferreira, P. C. (2006). Nova Terminologia Linguística para os Ensinos Básico e Secundário: Roteiros para a ação didática no Secundário. Porto: ASA.

Mateus, M. H. M., Pereira, D. \& Fischer, G. (2008). Diversidade Linguística na Escola Portuguesa. Projecto do ILTEC - Instituto de Lingüística Teórica e Computacional. Edição da Fundação Calouste Gulbenkian.

Montanero, M., Lucero, M., \& Fernández, M. J. (2014). Iterative co-evaluation with a rubric of narrative texts in Primary Education/Coevaluación iterativa con rúbrica de textos narrativos en la Educación Primaria. Infancia y Aprendizaje, 37(1), 184-220. doi.org/10.1080/02103702.2014.881653

Nicole, D., \& Macfarlane-Dick, D. (2006). Formative assessment and self-regulated learning: model and seven principles of good feedback. Studies in Higher Education, 34, 199-218.

Ortega, J. L. G. (2013). Cómo estructuran el contenido de la escritura alumnos de educación primaria. Contextos Educativos. Revista de Educación, (15), 9-26. doi.org/10.18172/con.652

Panadero, E., \& Jonsson, A. (2013). The use of scoring rubrics for formative assessment purposes revisited: A review. Educational Research Review, 9, 129-144.

Pereira, M.L.A. (2000). Escrever em Português Didácticas e Práticas. Porto: Edições Asa.

Pinheiro, A. (2007). O Desenvolvimento da Capacidade de Elaboração Escrita: Editorial Novembro

Pritchard, R. J., \& Honeycutt, R. L. (2006). The process approach to writinginstruction: Examining its effectiveness. Handbook of writing research, 275-290.

Ramos, J. L., Cuadrado, I., \& Iglesias, B. (2005). La composición escrita en el alumnado de Educación Primaria y Secundaria. Cultura y educación, 17(3), 239-251.

Raven, J., \& Raven, J. C. (1998). Jh Court. Manual for Raven's Progressive Matrices and Vocabulary Scales. Section 2: The Coloured Progressive Matrices.

Redondo, R. F., Sánchez, J. N. G., Torrance, M., \& Ramón, P. R. (2009). Cómo enseñar composición escrita en el aula: un modelo de instrucción cognitivo-estratégico y autorregulado. Aula Abierta, 37(1), 105-116.

Rust, C., Price, M., \& O'Donovan, B. E. (2003). Improving students' learning by developing their understanding of assessment criteria and processes. Assessment \& Evaluation in Higher Education, 28(2), 147-164. doi.org/10.1080/02602930301671

Silva, A. C. (2013). O impacto da revisão na qualidade de composições de crianças do $4^{\circ}$ ano de escolaridade. Psicologia: Reflexão e Crítica, 26(1).

Sim-Sim, I. (1997). Avaliação da linguagem oral: Um contributo para o conhecimento do descobrimento linguístico das crianças portuguesas. Textos de Educação. Lisboa: Fundação Calouste Gulbenkian.

Schunk, D. H., \& Zimmerman, B. J. (2007). Influencing children's self-efficacy and self-regulation of reading and writing through modeling. Reading and Writing Quarterly, 23, 7-25. doi.org/10.1080/10573560600837578
Torrance, M., \& Galbraith, D. (2006). The processing demands of writing. In C. A. MacArthur, S. Graham, \& J. Fitzgerald (Eds.), Handbook of writing research (pp. 67-80). New York: Guilford Press

Torrance, M., \& Jeffery, G. (1999). The cognitive demands of writing. Amsterdam, Netherlands: Amsterdam University Press.

Zimmerman, B. J., \& Risemberg, R. (1997). Becoming a self-regulated writer: A social cognitive perspective. Contemporary educational psychology, 22(1), 73-101. doi.org/10.1006/ceps.1997.0919

Zimmerman, B.J. (2002). Becoming a self regulated learner: an overview. Theory into Practice, 41, (2),64-70. doi:10.1207/s15430421 tip4102_2 